\title{
Ontogenia de los linfocitos B
}

\author{
Juan Carlos Balandrán ${ }^{1,2}$, Rosana Pelayo ${ }^{1}$
}

\section{Resumen}

El desarrollo de los linfocitos B a partir de células troncales hematopoyéticas es un proceso altamente regulado y continuo en el que se pierden gradualmente los potenciales de diferenciación múltiple y se adquieren funciones especializadas del linaje. A 50 años de su descubrimiento, el conocimiento actual de la diferenciación temprana de las células B proviene, en gran medida, del aislamiento y caracterización de los progenitores en la médula ósea que dan inicio al programa linfoide y de la definición de patrones de actividad transcripcional que controlan las decisiones de los destinos celulares. De especial relevancia ha sido la intercomunicación de los precursores con los componentes del microambiente hematopoyético para la generación de nuevos modelos que integren todos los elementos de regulación de este complejo proceso y para la comprensión de esta rama del sistema inmunológico adaptativo en la enfermedad. Esta revisión ofrece un panorama general del complejo proceso de diferenciación linfoide: la organización jerárquica y características biológicas de las células primitivas que participan en sus etapas más tempranas, hasta los principios que rigen su interdependencia con el microambiente hematopoyético.

PALABRAS CLAVE: linfocitos B, progenitores linfoides tempranos, médula ósea, microambiente hematopoyético, diferenciación linfoide.

Rev Alerg Méx 2016 Jan-Mar;63(1):71-79.

\section{B lymphocyte ontogeny.}

\author{
Juan Carlos Balandrán ${ }^{1,2}$, Rosana Pelayo ${ }^{1}$
}

\begin{abstract}
The B cell development from hematopoietic stem cells is a continuous and highly regulated process where multiple differentiation potentials are gradually lost while acquiring lineage specialized functions. At 50 years of the B cell discovery, the current knowledge of its early differentiation largely derive from the isolation and characterization of bone marrow early progenitor cells initiating the lymphoid program, and from the definition of transcriptional activity patterns that control cell fate decisions. Of particular relevance has been the intercommunication between B cell precursors and key components of the hematopoietic microenvironment, both for generation of novel models integrating all regulatory elements of this complex process, and for the understanding of this branch of the adaptive immune system in disease settings. This review provides an overview of the complex process of lymphoid differentiation: from the hierarchical organization and biological characteristics of primitive cells involved in its earliest stages, to the principles governing its interdependence with the hematopoietic microenvironment.
\end{abstract}

KEYWORDS: B lymphocytes; early lymphoid progenitors; bone marrow; hematopoietic microenvironment; lymphoid differentiation

\begin{abstract}
1 Laboratorio de Linfopoyesis, Unidad de Investigación Médica en Enfermedades Oncológicas, Coordinación de Investigación en Salud, Hospital de Oncología, Instituto Mexicano del Seguro Social, Ciudad de México.

${ }^{2}$ Departamento de Biomedicina Molecular, Centro de Investigación y de Estudios Avanzados del Instituto Politécnico Nacional, Ciudad de México.
\end{abstract}

Recibido: 12 de octubre 2015

Aceptado: 18 de enero 2016

Correspondencia

Dra. Rosana Pelayo

rosanapelayo@gmail.com

Este artículo debe citarse como

Balandrán JC, Pelayo R. Ontogenia de los linfocitos B. Rev Alerg Méx. 2016 ene-mar;63(1):71-79. 


\section{ANTECEDENTES}

A lo largo de la historia, el campo de la inmunología del desarrollo de los linfocitos B ha progresado paralelamente, aunque en dirección opuesta al de la biología de las células troncales hematopoyéticas. Mientras que en el primer caso la búsqueda de los progenitores tempranos en la médula ósea evolucionó a partir de los hallazgos funcionales en las células maduras, en el segundo, la identificación de las células seminales determinó el establecimiento de los principios básicos de diferenciación de los sistemas complejos y la construcción de modelos hematopoyéticos jerárquicos basados en estadios críticos de diferenciación, compromiso y maduración de los progenitores y precursores. Así, el conocimiento generado en la interfase entre la inmunología y la hematología ha tenido la más notable contribución en la ontogenia de los linfocitos B.

En 1965 se reconoció la conspicua población de células B como un linaje hematopoyético linfoide distinto, con un papel central en la respuesta inmunitaria adaptativa y características funcionales únicas, ${ }^{1,2}$ pero no fue hasta 32 años después cuando la clonación del progenitor linfoide común de ratón dio inicio a la elucidación de su origen celular. ${ }^{3}$ Los años intermedios fueron clave en la determinación de sus cinéticas de proliferación y el descubrimiento de la composición y mecanismos moleculares de expresión de sus receptores de antígeno (BCR), ${ }^{4-6}$ en tanto que los subsecuentes establecieron que la enzima recombinasa RAG1 marca el inicio del programa de diferenciación linfoide..$^{7-9}$ La recombinación somática, así como las cascadas de señalización implicadas en la activación linfocitaria, fueron piedras angulares para ambas piezas del conocimiento. $6,10,11$

\section{Los primeros pasos hacia el compromiso linfoide}

La hematopoyesis es el proceso de generación de los más de 10 tipos celulares del tejido san- guíneo. La demostración en 1961 de su origen clonal en una célula troncal ${ }^{12-15}$ y posteriormente de las propiedades biológicas que definen a esta célula, autorrenovación y potencial de diferenciación múltiple, establecieron el paradigma de la diferenciación celular a partir de sistemas altamente complejos y organizados. ${ }^{11,15,16}$ Por un lado, la identificación fenotípica de las células troncales hematopoyéticas en el hígado fetal y en la médula ósea adulta del ratón, ${ }^{17}$ y por otro, la intensa explotación de modelos experimentales para el estudio de su potencial y su regulación impulsaron la construcción de mapas jerárquicos de diferenciación. En ellos, el desarrollo linfopoyético temprano es guiado por combinaciones de factores intrínsecos y microambientales que impulsan la pérdida gradual de opciones de diferenciación en paralelo con una ganancia de funciones especializadas. ${ }^{8,9,11} \mathrm{Al}$ menos cinco grandes compartimientos se han identificado en esos mapas: el de las células troncales, el de los progenitores multipotenciales, el de los progenitores oligopotenciales, el de los precursores y el de las células maduras (Figura 1).

El primer compartimiento corresponde a las células más primitivas: las células troncales hematopoyéticas. Su aptitud clonal para reconstituir a largo plazo el sistema hematopoyético de animales de experimentación irradiados e inmunodeficientes les merece el nombre de LTHSC (long term hematopoietic stem cells). En el ratón, éstas residen en la fracción LSK (por su fenotipo Lin-Sca $1^{+} \mathrm{C}-\mathrm{kit}^{\mathrm{hi}}{ }^{\mathrm{i}}$, constituyendo aproximadamente $0.1 \%$ del total celular de la médula ósea, en donde coexisten todas las poblaciones que carecen de expresión de marcadores de linajes maduros, que incluyen CD3, CD8, CD19, CD20, NK1.1, CD11b, CD14 o Ter119.8,9,16 En humanos, el antígeno CD34 ha resultado el principal marcador para las células troncales hematopoyéticas y los progenitores multi y oligopotenciales. De las células hematopoyéticas en médula ósea humana, 0.5-5\% expresan CD34 y sólo una pequeña fracción (1-10\%) de 


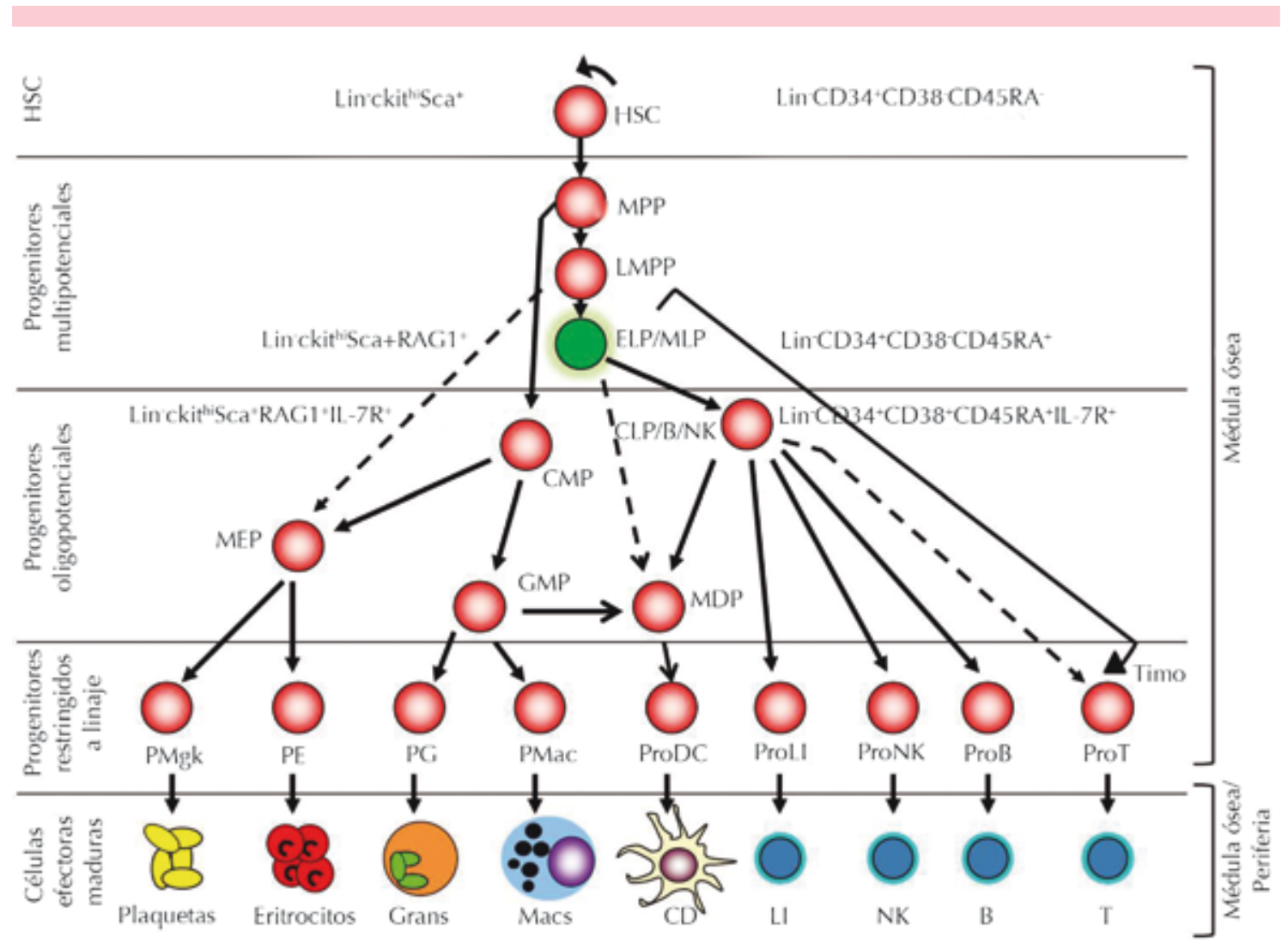

Figura 1. Ontogenia de los linfocitos B en el contexto jerárquico de diferenciación hematopoyética. La hematopoyesis inicia en una célula troncal, a partir de la que se diferencian de manera gradual y continua los progenitores que restringen su compromiso de linaje. El mapa muestra los cinco compartimentos que componen el sistema hematopoyético, así como las características fenotípicas más relevantes de las subpoblaciones en diferenciación provenientes de ratón (izquierda) y humano (derecha).

HSC: célula troncal hematopoyética; MPP: progenitor multipotencial; LMPP: progenitor multipotencial predispuesto al linaje linfoide; ELP: progenitor linfoide temprano; MLP: progenitor multilinfoide; CLP: progenitor linfoide común; B/NK: progenitor de células B y NK; CMP: progenitor mieloide común; MEP: progenitor de megacariocitos y eritrocitos; GMP: progenitor granulocítico y monocítico; MDP: progenitor de macrófagos y células dendríticas; LI: linfocitos innatos.

Modificada de las referencias 14 y 15 .

las células Lin-CD34+CD38- está enriquecida en células troncales con capacidad de reconstituir a largo plazo el sistema hematopoyético (Figura 1). ${ }^{15}$ Estas células expresan, además, antígenos como CD90, CD117, CD133 y VEGFR2, que las distinguen de células en estadios de diferenciación más avanzados. De manera notable, el descubrimiento y caracterización biológica de las células troncales hematopoyéticas ha trascendido en tal magnitud que su uso para la regeneración de tejidos y el rescate funcional en enfermedades hematopoyéticas e inmunológicas ha revolucionado la Medicina moderna.

Las células troncales hematopoyéticas dan origen a los progenitores multipotenciales, que 
conforman el segundo compartimento y el más heterogéneo del sistema. En éste, los progenitores han perdido la capacidad de autorrenovación y la de reconstitución a largo plazo. En el ratón, los estadios de transición progenitor multipotencial predispuesto al linaje linfoide y progenitor linfoide temprano (early lymphoid progenitors) son el inicio del programa de diferenciación linfoide, evidente por la expresión de la enzima recombinasa RAG1. Sus contrapartes en el humano aparentemente residen en la fracción de progenitores multilinfoides (multi-lymphoid progenitors). Río abajo, la vía avanza hacia los progenitores oligopotenciales, que han incrementado su capacidad de proliferación, pero sus potenciales de diferenciación se restringen a dos o tres tipos celulares. Aunque comparten ciertas características fenotípicas con las células de los compartimentos anteriores, estos progenitores tienen patrones de expresión antigénica de acuerdo con el programa de diferenciación que inicia (Figura 1). A pesar de su inmadurez, las células que residen en el acervo de precursores unipotenciales son altamente proliferativas y reconocibles por su morfología a través de microscopia de luz. Este compartimento contiene más de $90 \%$ de las células hematopoyéticas residentes en la cavidad de la médula ósea. ${ }^{15}$ Por último, las células terminalmente diferenciadas y en vías de maduración son morfológica, fenotípica y funcionalmente distintas y se dividen en dos grandes grupos: mieloides y linfoides. Las primeras comprenden a los granulocitos (neutrófilos, basófilos y eosinófilos), monocitos, macrófagos, eritrocitos, megacariocitos, células cebadas y células dendríticas mieloides, mientras que las segundas comprenden a los linfocitos B, los linfocitos $T$ y diversas subpoblaciones de células innatas, incluidas las células NK, las células linfoides innatas (o linfocitos innatos) y algunas categorías de células dendríticas de origen linfoide. Las células de linaje mieloide son producidas a través de un proceso dinámico conocido como mielopoyesis, en tanto que las de linaje linfoide son consecuencia de la linfopoyesis.
Progenitores linfoides de células $B$ : luz a través de los ratones RAG1-GFP

La población de progenitores multipotenciales predispuestos al linaje linfoide contiene progenitores clonales con un potencial combinado de células $\mathrm{B}$, T y mieloide, ${ }^{18}$ así como restringidos a linajes de B o T, y productores de células NK. La regulación negativa de la molécula de adhesión VCAM- ${ }^{19}$ y la transcripción del locus de la enzima que recombina los segmentos genéticos VDJ de la inmunoglobulina y del TCR, la recombinasa RAG1, marcan a las células que apenas inician el programa de diferenciación hacia la estirpe linfoide.? Por ello, los ratones knockin RAG1/GFP se constituyeron como una herramienta particularmente útil en la investigación del proceso de linfopoyesis temprana, ,,16,20-22 y permitieron el aislamiento y caracterización de los progenitores linfoides más tempranos en el hígado fetal y en médula ósea de los que se tiene conocimiento: los progenitores linfoides tempranos. En ese modelo, un alelo del gen RAG1 se reemplazó por la secuencia que codifica la proteína verde fluorescente y fue posible localizar la señal de fluorescencia por análisis de citometría de flujo, que corresponde con la transcripción del gen RAG1.7,20 Las células que expresan RAG-1 pueden clasificarse en una serie de estadios de diferenciación, comenzando con la fracción ckithisca $1^{+}{ }^{-}$FPlo y culminando con subpoblaciones GFPhi. En el hígado fetal y en la médula ósea adulta, las células troncales y los progenitores mieloides residen en la fracción GFP-, mientras que los progenitores linfoides en la $\mathrm{GFP}^{+}$. Los progenitores linfoides tempranos son parte de la población LSK, de fenotipo Lin' ${ }^{-}$ckithiSca-1+Thy 1.1 $^{+}$ IL7-R $-\mathrm{TdT}{ }^{+} \mathrm{CD} 27^{+}{ }^{+} \mathrm{Flt} 3{ }^{+}$y altamente sensibles al tratamiento con estrógenos. ${ }^{7,16,23} \mathrm{Su}$ potencial para generar todas las líneas de células linfoides es muy alto, así como para producir células del sistema inmunológico innato linfoide, pero el potencial de diferenciación mieloide es reducido. Acorde con estas características, los progenitores linfoides tempranos transcriben genes asociados 
con linajes linfoides como gata-3, ebf, b29 y dan origen a la fracción de pro-linfocitos Lin-ckit ${ }^{\text {loS }}$ Ca$1^{+}$Thy $1.1-$ Flt $^{+}{ }^{+} \mathrm{GFP}^{+}$, que desregula la expresión de c-kit e incluye a la mayor parte de los progenitores linfoides comunes o CLP (common lymphoid progenitors). La participación de estos progenitores está marcada por la expresión en superficie del receptor de IL-7 (IL-7R) y su distintiva señalización por STAT5, y aunque tienen cierta actividad clonogénica de T, B y NK (lo que originalmente les valió su designación), se reconocen como los más eficientes precursores de linfocitos B. ${ }^{24}$

\section{De la "fracción A" al BCR y la migración periférica}

Otro parteaguas en la construcción del mapa de diferenciación linfoide se estableció con los trabajos de Hardy, en 1991, en los que se identificaron las principales subpoblaciones con participación de linaje $B$ en la médula ósea y se propuso un esquema secuencial de diferenciación río abajo de los progenitores linfoides comunes. ${ }^{25,26} \mathrm{El}$ compartimento celular más primitivo exhibía un fenotipo $\mathrm{B} 22 \mathrm{O}^{+} \mathrm{CD} 19^{-}$ $\mathrm{CD} 43^{+} \mathrm{CD} 24^{-/ \mathrm{lo}}$ y se denominó fracción A. Esta fracción comprende tres subpoblaciones definidas no yuxtapuestas: DX5-Ly6C+ $(45-55 \%)$, DX5+'Ly6C- (30-35\%) y DX5-'-Ly6C- (16-20\%), de las que sólo la última tiene potencial precursor de células $B^{16,27,28}$ y se denominó pre-proB. La caracterización reciente de células de linaje no-B dentro de las otras dos subpoblaciones (DX5Ly $6 C^{+}$y DX $5^{+}$Ly $\left.6 C^{-}\right)$y sus controvertidos orígenes incrementaron la complejidad del sistema linfohematopoyético. Dos categorías de células dendríticas plasmacitoides $(\mathrm{pDC})$ residen entre las DX5-Ly6C+ de la fracción A que expresan $\mathrm{CD} 11 \mathrm{c},{ }^{29}$ mientras que la subpoblación cohorte DX5+Ly6C- contiene células que coexpresan el marcador NK1.1 y que se identifican como IKDC (interferon-producing killer dendritic cells) con poderosa capacidad citotóxica y antitumoral, así como de producción de IFN- $\gamma \cdot{ }^{22}$
En conclusión, parte de la fracción A corresponde a células del sistema inmunológico innato, como las pDCs, NKs e IKDCs, que tienen su origen en progenitores linfoides tempranos y cuyo brazo de diferenciación aparentemente es previo e independiente de la participación de los precursores en la ruta de células B pre-proB. Hay grandes expectativas acerca de la posible inclusión de otras células linfoides innatas de reciente descubrimiento dentro de la fracción A de Hardy.

La regulación positiva de CD19 es uno de los sellos más tempranos del compromiso del linaje $B$, que participa en la transducción de señales de las células en el proceso de diferenciación. Las células denominadas pre-proB residentes en la fracción A2 de Hardy expresan IgH en línea germinal, además de genes que codifican para componentes de los receptores pre-B y B, como mb-1, B29 y I5, y factores de transcripción necesarios para la diferenciación, como Pax5 y E47 (Figura 2). Las señales de IL-7 son críticas en esta transición, como lo indica el bloqueo profundo en el desarrollo de $B$ a nivel de la fracción A2 de la médula ósea de ratones deficientes en IL-7R..$^{29-31}$ Los subsecuentes estadios independientes de antígeno son guiados con la finalidad de expresar moléculas de inmunoglobulina funcionales en membrana $y$, de manera orquestada, diversos factores de transcripción están implicados en esta ruta (Figura 2). De ellos, PU.1, Ikaros, E2A, Bcl11a, EBF y Pax5 participan en la determinación del compromiso, la especificación del linaje o ambos. La deficiencia de PU.1 resulta en que Flt3 y EBF no sean expresados, bloqueando la diferenciación de células B. ${ }^{32}$ E2A es decisivo para la activación de RAG, y sus productos, directa o indirectamente, regulan la expresión de Pax5, que a su vez regula la expresión de genes específicos de células B, incluyendo CD19. ${ }^{11,16}$ Además, un número de genes que participan en la señalización del receptor pre-B, como mb-1, ë $\lambda 5$, VpreB y B29, así 


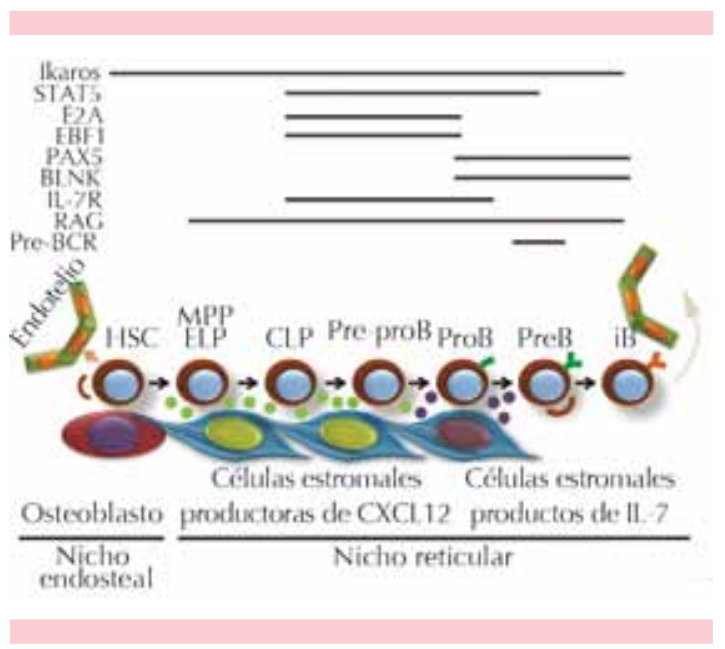

Figura 2. Regulación intrínseca y microambiental del desarrollo temprano del linaje B. En la médula ósea, la transición de los estados de diferenciación linfoide B depende de los programas genéticos en concordancia con señales proporcionadas por el microambiente hematopoyético. Las células estromales altamente productoras de CXCL12 conforman los nichos linfoides tempranos, en tanto que su contraparte productora de IL-7 sostiene las etapas precursoras proB. Las células preB adquieren independencia de IL-7 concomitante a la formación del pre-BCR. La producción de células B inmaduras va acompañada de su migración a la periferia. Se muestran los factores de transcripción que participan más activamente en la vía de diferenciación de linfocitos B.

HSC: célula troncal hematopoyética; MPP: progenitor multipotencial; ELP: progenitor linfoide temprano; CLP: progenitor linfoide común.

Modificada de la referencia 11.

como RAG1/2 y TdT requeridos para el rearreglo de inmunoglobulinas, son blancos potenciales para las secuencias de unión a ADN de E2A. ${ }^{33}$ EBF comienza su expresión en los progenitores linfoides tempranos y en coordinación con los productos de E2A, EBF es clave en el control previo al rearreglo de genes de inmunoglobulina. ${ }^{32}$

Por último, la función de Pax5 es esencial para la represión de la transcripción de genes no linfoides y no B. ${ }^{34,35}$ Entonces, en esta red de regulación, el desarrollo de progenitores multipotentes es dependiente de PU.1 e Ikaros, mientras que la especificación y el compromiso de las células pro-B son dependientes de E2A/ EBF y Pax5.

En humanos, a partir del progenitor de células $B$ y NK (B/NK) se derivan las células pro-B con fenotipo $\mathrm{CD} 34^{+} \mathrm{CD} 19^{+} \mathrm{CD} 10^{+}$y de ahí secuencialmente las pre- $\mathrm{BI}$ grandes $\mathrm{CD} 34^{+} \mathrm{CD} 19^{+} \mathrm{CD} 10^{+}$, pre-BII grandes $\mathrm{CD} 34{ }^{-} \mathrm{CD} 19^{+} \mathrm{CD} 10^{+}$, pre-BII pequeñas $\mathrm{CD} 34^{-} \mathrm{CD} 19^{+} \mathrm{CD} 10^{+}$, B inmaduras CD34-CD19+CD10+slgM ${ }^{+}$hasta la producción de $\mathrm{B}$ maduras CD34-CD19+CD10-slgM ${ }^{+}$slgD ${ }^{+}$, que a la larga se exportarán a los tejidos linfoides periféricos para cumplir su función de reconocimiento de antígeno, activación y producción de anticuerpos específicos. ${ }^{15}$ La linfopoyesis de $B$ en humanos parece cumplirse sin el estricto requerimiento de la IL-7.

\section{El microambiente hematopoyético en la regulación de la linfopoyesis de $B$}

Las células primitivas y las terminalmente diferenciadas crecen en estrecha comunicación con el microambiente de la médula ósea y en exposición a combinaciones y concentraciones variables de citocinas. ${ }^{15}$ La contribución de células estromales, osteoblastos y células endoteliales en los distintos estadios de diferenciación linfo-hematopoyética es decisiva. ${ }^{36-38} \mathrm{EI}$ microambiente especializado que sostiene a las células troncales constituye el nicho hematopoyético que promueve el mantenimiento de las células troncales hematopoyéticas a lo largo de la vida. Se propone que para que un microambiente se considere nicho deben satisfacerse dos criterios: que en él resida la célula progenitora in vivo y que promueva su mantenimiento. A la luz del conocimiento actual existen al menos tres tipos de nichos: el osteoblástico, el vascular y el reticular. El primero de ellos está en el endosteo y da soporte a través de los osteoblastos 
a las células troncales hematopoyéticas. ${ }^{38}$ Los osteoblastos proveen señales reguladoras de la quiescencia a la población de células troncales hematopoyéticas a través de la vía no canónica de Wnt, angiopoyetina, trombopoyetina, SDF-1/ CXCL12 y osteopontina. Por su parte, el nicho vascular reside alrededor de los sinusoides y a través de su endotelio las células hematopoyéticas pueden entrar y salir de la circulación. Este nicho es aparentemente inductor. En el lumen de la médula ósea tiene lugar la mayor parte de los procesos de diferenciación linfoide temprana. El ambiente perivascular está marcado por células reticulares altamente productoras de CXCL12 y de IL-7 que promueven la diferenciación de los precursores B (Figura 2). Además, otras células, como las mesenquimales con potencial para generar hueso, cartílago y grasa, son reguladores esenciales de la hematopoyesis normal. ${ }^{37,38} \mathrm{El}$ nicho endosteal CXCL12+ Nestina+, y posiblemente el endotelial, mantienen a las células troncales hematopoyéticas en ciclos de prolongada quiescencia en los que alrededor de $70 \%$ de las células están en $\mathrm{G}_{0^{\prime}}$ presumiblemente debido a la expresión de factores genéticos intrínsecos que inhiben el ciclo celular, como p21 y pTEN, así como a la actividad de diversas moléculas de anclaje. ${ }^{39-41}$ Diversos estudios indican que la mayor parte de los progenitores linfoides tempranos pasan también un tiempo considerable en $\mathrm{G}_{0}{ }^{15}$ en nichos de células estromales CXCL12+, Ilamadas CAR (CXCL12-abundant reticular cells $)^{42,43}$ y que esta condición quiescente puede ser importante en el control del tamaño de la población y para la integridad de las células que reabastecen el sistema inmunológico a lo largo de la vida. ${ }^{16}$ De especial importancia es la estrecha comunicación y dependencia del estadio temprano pre-proB y el más tardío de células plasmáticas a las células CAR, mientras que en la etapa proB la dependencia está relacionada con células estromales que producen IL-7 (Figura 2). La relevante investigación de Nagasawa y colaboradores sugiere entonces que en la médula ósea las células CAR constituyen los nichos del desarrollo de células B y que la investigación del papel de CXCL12 en la patobiología de las enfermedades de este linaje -leucemias, linfomas y mielomas - será prioridad de la investigación biomédica en los años venideros. Para lograrlo, debe considerarse que la arquitectura tridimensional de los nichos es decisiva para la adecuada regulación del proceso hematopoyético, por lo que existe un especial interés por el desarrollo y aplicación de modelos de estudio tridimensionales (3D), así como la construcción de organoides que promuevan las interacciones que inician y mantienen los procesos de diferenciación de los precursores $\mathrm{B}$, tanto en la normalidad como en emergencia hematopoyética.

\section{Perspectivas}

Por su importancia para la práctica clínica, posiblemente una de las prioridades del mundo biomédico será definir los mecanismos que perturban los patrones de diferenciación temprana de las células B en circunstancias patológicas de inflamación, autoinmunidad, inmunodeficiencias y cáncer. Nuevas y poderosas plataformas de estudio, experimentales y teóricas, que consideren la complejidad de la intercomunicación hematopoyéticamicroambiental, serán indispensables para el cumplimiento de esta labor.

\section{Agradecimientos}

Los autores agradecen el financiamiento de sus investigaciones acerca de Células troncales y diferenciación linfoide proveniente de la Coordinación de Investigación en Salud del IMSS y del Consejo Nacional de Ciencia y Tecnología.

\section{REFERENCIAS}

1. Cooper MD. The early history of B cells. Nature Reviews Immunology 2015;15:191-197. 
2. Cooper MD, Peterson RD, Good RA. Delineation of the Thymic and bursal lymphoid systems in the chicken. Nature 1965;205:143-146

3. Kondo M, Weissman IL, Akashi K. Identification of clonogenic common lymphoid progenitors in mouse bone marrow. Cell 1997;91:661-672.

4. Kincade PW, Lawton AR, Cooper MD. Restriction of surface immunoglobulin determinants to lymphocytes of the plasma cell line. J Immunol 1971;106:1421-1423.

5. Osmond DG, Nossal GJ. Differentiation of lymphocytes in mouse bone marrow. II. Kinetics of maturation and renewal of antiglobulin-binding cells studied by double labeling. Cel Immunol 1974;13:132-145.

6. Sakano H, Maki R, Kurosawa Y, Roeder W, Tonegawa S. Two types of somatic recombination are necessary for the generation of complete immunoglobulin heavy-chain genes. Nature 1980;286:676-683.

7. Igarashi H, Gregory SC, Yokota T, Sakaguchi N, Kincade PW. Transcription from the RAG1 locus marks the earliest lymphocyte progenitors in bone marrow. Immunity 2002;17:117-130.

8. Pelayo R, Welner R, Perry SS, Huang J, et al. Lymphoid progenitors and primary routes to becoming cells of the immune system. Current Opin Imunol 2005;17:100-107.

9. Baba Y, Pelayo R, Kincade PW. Relationships between hematopoietic stem cells and lymphocyte progenitors. Trends Immunol 2004;25:645-649.

10. Reth $\mathrm{M}$, Nielsen P. Signaling circuits in early B-cell development. Advances in Immunology 2014;122:129-175.

11. Pelayo R, Dorantes-Acosta E, Vadillo E, Fuentes-Panana EM. From HSC to B-lymphoid cells in normal and malignant hematopoiesis: InTech, 2012.

12. Till JE, Mc CE. A direct measurement of the radiation sensitivity of normal mouse bone marrow cells. Radiation Research 1961;14:213-222.

13. Dick JE. Stem cell concepts renew cancer research. Blood 2008;112:4793-4807.

14. Seita J, Weissman IL. Hematopoietic stem cell: self-renewal versus differentiation. Wiley Interdiscip Rev Syst Biol Med 2010;2:640-653.

15. Vadillo E, Pelayo R. El sistema hematopoyético a partir de células troncales. In: Pelayo R, editor. Células Troncales y Medicina Regenerativa. Mexico: PUIS, 2011;143-171.

16. Welner RS, Kincade PW, Pelayo R. Linfopoyesis temprana en médula ósea adulta. Inmunología 2007;26:135-144.

17. Spangrude GJ, Heimfeld S, Weissman IL. Purification and characterization of mouse hematopoietic stem cells. Science 1988;241:58-62.

18. Mansson R, Hultquist $A$, Luc $S$, Yang $L$, et al. Molecular evidence for hierarchical transcriptional lineage priming in fetal and adult stem cells and multipotent progenitors. Immunity 2007;26:407-419.
19. Lai AY, Lin SM, Kondo M. Heterogeneity of Flt3-expressing multipotent progenitors in mouse bone marrow. J Immunol 2005;175:5016-5023

20. Yokota $\mathrm{T}$, Kouro $\mathrm{T}$, Hirose J, Igarashi $\mathrm{H}$, et al. Unique properties of fetal lymphoid progenitors identified according to RAG1 gene expression. Immunity 2003;19:365-375.

21. Perry SS, Welner RS, Kouro T, Kincade PW, Sun XH. Primitive lymphoid progenitors in bone marrow with $\mathrm{T}$ lineage reconstituting potential. J Immunol 2006;177:2880-2887.

22. Welner RS, Pelayo R, Garrett KP, Chen X, et al. Interferonproducing killer dendritic cells (IKDCs) arise via a unique differentiation pathway from primitive c-kitHiCD62L+ lymphoid progenitors. Blood 2007;109:4825-4931.

23. Medina KL, Garrett KP, Thompson LF, Rossi MI, et al. Identification of very early lymphoid precursors in bone marrow and their regulation by estrogen. Nature Immunology 2001;2:718-724.

24. Kondo M, Wagers AJ, Manz MG, Prohaska SS, et al. Biology of hematopoietic stem cells and progenitors: implications for clinical application. Ann Rev Immunol 2003;21:759-806.

25. Hardy RR, Carmack CE, Shinton SA, Kemp JD, Hayakawa $K$. Resolution and characterization of pro-B and pre-pro$B$ cell stages in normal mouse bone marrow. J Exp Med 1991;173:1213-1225.

26. Rumfelt LL, Zhou Y, Rowley BM, Shinton SA, Hardy RR. Lineage specification and plasticity in CD19- early $B$ cell precursors. J Exp Med 2006;203:675-687.

27. Li YS, Wasserman R, Hayakawa K, Hardy RR. Identification of the earliest $B$ lineage stage in mouse bone marrow. Immunity 1996;5:527-535.

28. Tudor KS, Payne KJ, Yamashita Y, Kincade PW. Functional assessment of precursors from murine bone marrow suggests a sequence of early $B$ lineage differentiation events. Immunity 2000;12:335-345.

29. Pelayo R, Hirose J, Huang J, Garrett KP, et al. Derivation of 2 categories of plasmacytoid dendritic cells in murine bone marrow. Blood 2005;105:4407-4415.

30. Allman D, Li J, Hardy RR. Commitment to the B lymphoid lineage occurs before $\mathrm{DH}-\mathrm{JH}$ recombination. J Exp Med 1999;189:735-740.

31. Pelayo R, Welner RS, Nagai Y, Kincade PW. Life before the pre-B cell receptor checkpoint: specification and commitment of primitive lymphoid progenitors in adult bone marrow. Semin Immunol 2006;18:2-11.

32. Medina KL, Pongubala JM, Reddy KL, Lancki DW, et al. Assembling a gene regulatory network for specification of the B cell fate. Develop Cell 2004;7:607-617.

33. Sun XH. Multitasking of helix-loop-helix proteins in lymphopoiesis. Adv Immunol 2004;84:43-77.

34. Nutt SL, Heavey B, Rolink AG, Busslinger M. Commitment to the B-lymphoid lineage depends on the transcription factor Pax5. Nature 1999;401:556-562. 
35. Busslinger M. Transcriptional control of early B cell development. Ann Rev Immunol 2004;22:55-79.

36. Blom B, Spits $H$. Development of human lymphoid cells. Ann Rev Immunol 2006;24:287-320.

37. Pelayo R, Miyazaki K, Huang J, Garrett KP, et al. Cell cycle quiescence of early lymphoid progenitors in adult bone marrow. Stem Cells 2006;24:2703-2713.

38. Passegue E, Wagers AJ, Giuriato S, Anderson WC, Weissman IL. Global analysis of proliferation and cell cycle gene expression in the regulation of hematopoietic stem and progenitor cell fates. J Exp Med 2005;202:1599-1611.

39. Sugiyama T, Kohara H, Noda M, Nagasawa T. Maintenance of the hematopoietic stem cell pool by CXCL12-CXCR4 chemokine signaling in bone marrow stromal cell niches. Immunity 2006;25:977-988.

40. Ehninger A, Trumpp A. The bone marrow stem cell niche grows up: mesenchymal stem cells and macrophages move in. J Exp Med 2011;208:421-428.

41. Suda T, Arai F, Hirao A. Hematopoietic stem cells and their niche. Trends Immunol 2005;26:426-433.

42. Nagasawa T. CXCL12/SDF-1 and CXCR4. Frontiers Immunol 2015;6:301.

43. Nagasawa T. Microenvironmental niches in the bone marrow required for B-cell development. Nature Rev Immunol 2006;6:107-116. 